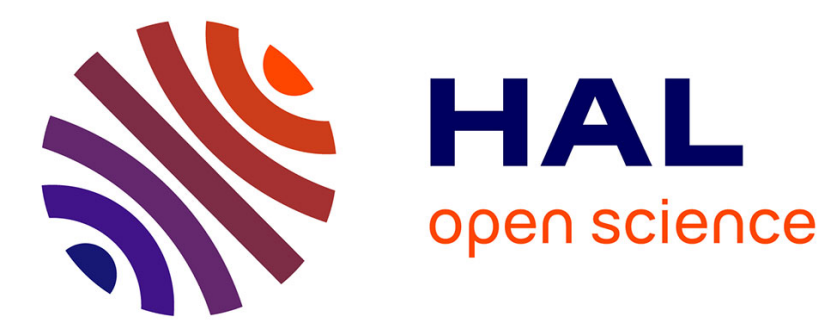

\title{
Alignment of atomic inner-shells following alpha-decay-induced ionization
}

S R Mcconnell, a N Artemyev, A Surzhykov

\section{To cite this version:}

S R Mcconnell, a N Artemyev, A Surzhykov. Alignment of atomic inner-shells following alpha-decayinduced ionization. Journal of Physics B: Atomic, Molecular and Optical Physics, 2011, 44 (14), pp.145204. 10.1088/0953-4075/44/14/145204 . hal-00636643

\section{HAL Id: hal-00636643 \\ https://hal.science/hal-00636643}

Submitted on 28 Oct 2011

HAL is a multi-disciplinary open access archive for the deposit and dissemination of scientific research documents, whether they are published or not. The documents may come from teaching and research institutions in France or abroad, or from public or private research centers.
L'archive ouverte pluridisciplinaire HAL, est destinée au dépôt et à la diffusion de documents scientifiques de niveau recherche, publiés ou non, émanant des établissements d'enseignement et de recherche français ou étrangers, des laboratoires publics ou privés. 


\title{
Alignment of atomic inner-shells following alpha-decay induced ionisation
}

\author{
S R McConnell ${ }^{1,2}$, A N Artemyev ${ }^{1,2}$ and A Surzhykov ${ }^{1,2}$ \\ ${ }^{1}$ Physikalisches Institut der Universität Heidelberg, Philosophenweg 12, 69020 \\ Heidelberg, Germany \\ ${ }^{2}$ GSI Helmholtzzentrum für Schwerionenforschung GmbH, Planckstraße 1, 64291 \\ Darmstadt, Germany \\ E-mail: smcconne@physi.uni-heidelberg.de
}

\begin{abstract}
.
Nuclear alpha decay may induce the emission of electrons originally bound to the nucleus. If the ejected electron initially resides in a state with $j>1 / 2$, the residual ion may be aligned. In this article, such an alignment is examined for the $\mathrm{L}_{\mathrm{III}}$ ionisation of heavy atoms. This work pays particular attention to the effects of shake-off, recoil and nuclear potential screening. To illustrate these effects, we have performed calculations for the decay of the nuclei of neutral Polonium and Thorium atoms over a range of commonly encountered alpha particle kinetic energies $\left(\epsilon_{\alpha} \sim O(10 \mathrm{MeV})\right)$.
\end{abstract}

PACS numbers: 23.60.+e, 34.50.Fa, 23.20.-g., 27.80.+w, 27.90.+b

Submitted to: J. Phys. B: At. Mol. Phys. 


\section{Introduction}

The alpha decay of heavy nuclei and its effects on bound electrons has had a long tradition of study over the course of the last forty years [1, 2, 3, 4]. Within this field, attention has been paid predominantly to cross sections of inner shell electron ionisation. In contrast to the properties of the emitted electron, less focus has been given to the subject of the state of the residual atom. Information about the subsequent state can be gained by analysing the characteristic photon emission. The angular and polarisation properties thereof are related to the alignment of the holes produced in the inner atomic shells, i.e. the population of the sub-states $\left|j m_{j}\right\rangle$ where $j>1 / 2$.

To date the only study of alignment resulting from the alpha decay of heavy atoms was that conducted by Kabachnik [5]. In that work, the semi-classical approximation together with non-relativistic perturbation theory [6] was employed to estimate probabilities for the ionisation of Polonium where an electron, initially bound to the the $2 \mathrm{p}_{3 / 2}$ state, is excited into the continuum. However, a more accurate investigation of alignment may require an expansion of the theory applied in [5] to include relativistic wavefunctions, recoil and shake-off effects and the interaction of the neighbouring electrons. Apart from the effects of these phenomena, the realistic motion of the alpha particle should also be accounted for.

In this work we present a study of the alignment of the residual hole created by the alpha-decay induced ionisation of heavy atoms. We have implemented the effects of recoil, shake-off and potential screening and used relativistic wavefunctions in all calculations. To address the issue of alpha particle motion, we shall consider the atomic system using two ansatzes. The first of these approaches corresponds to that used in $[7,8]$, where the alpha particle appears at the co-ordinate origin and attains its final velocity suddenly. The second approach presented will make use of a realistic picture of alpha particle motion. In such a picture, after having tunnelled through the potential barrier, the alpha particle appears at a particular distance outside of the nucleus and moves away under Coulombic repulsion.

Within the context of these two types of alpha particle motion, we have chosen to adhere to the following structure in this article. In the next section the theoretical prerequisites for performing alignment calculations will be presented. From there a comparison will be made with the previous calculations performed in [5]. Furthermore, a comparison between the two scenarios of alpha particle motion will also be given. The theoretical approach presented is applicable to all heavy atom systems that are susceptible to alpha decay. We have chosen Polonium and Thorium as test cases in order to firstly compare with previous calculations conducted on Polonium, and secondly to illustrate any alignment dependence on the charge of the parent nucleus. All formulas are shown in natural units $\left(\hbar=c=m_{e}=1, \alpha=e^{2}\right)$. 


\section{Theory of atomic ionisation in alpha decay}

\subsection{Transition amplitudes}

The analysis of alpha decay induced atomic ionisation can be performed in the semiclassical approximation with first order perturbation theory. Under the semi-classical approximation, one treats the alpha particle as a classical particle moving along a straight trajectory, and the electron as a quantum particle, whose wavefunctions here are Dirac eigenfunctions. Under these assumptions, the transition amplitude $a_{f i}$ of the ionisation of an atom by electron emission is given by

$$
a_{f i}=\int_{-\infty}^{\infty} e^{i\left(E_{f}-E_{i}\right) t}\left\langle\psi_{f}\left|H^{1}\right| \psi_{i}\right\rangle d t
$$

where $E_{f},\left|\psi_{f}\right\rangle$ and $E_{i},\left|\psi_{i}\right\rangle$ are the energies and many-electron wavefunctions of the final and initial states before and after ionisation respectively. The evaluation of the amplitude (1) using many-electron wavefunctions is highly non-trivial, therefore we make use of the single active electron approximation. This is well justified for the description of inner-shell atomic processes if $\left|\psi_{i}\right\rangle$ and $\left|\psi_{f}\right\rangle$ are taken to be the bound and continuum state solutions of the Dirac equation for a screened potential. Further elaborating on equation (1), the final state wavefunction can be written as

$$
\begin{aligned}
\left|\psi_{f}\right\rangle & =\left|\mathbf{k} \mu_{s}\right\rangle \\
& =\sum_{\varkappa, \mu_{\jmath}} i^{\ell}\left(\ell, \mu_{\jmath}-\mu_{s}, \frac{1}{2}, \mu_{s} \mid \jmath, \mu_{\jmath}\right) e^{-i \Delta_{\varkappa}} Y_{\ell}^{\mu_{\jmath}-\mu_{s} *}(\hat{\mathbf{k}})\left|E_{f} \varkappa \mu_{\jmath}\right\rangle,
\end{aligned}
$$

where $\mathbf{k}$ is the momentum vector of the emitted electron. An expansion for $\left|E_{f} \varkappa \mu_{\jmath}\right\rangle$ and the initial state wavefunction $\left|\psi_{i}\right\rangle \equiv\left|n \kappa m_{j}\right\rangle$ in the analytic unscreened potential representation may be found in [9].

By adopting the alpha particle trajectory as the quantisation axis, we can write $H^{1}$ in the form $[1,2]$

$$
H^{1}(t)=\Theta(t)\left[-\frac{Z_{1} \alpha\left(1-\beta \alpha_{3}\right)}{\left|\mathbf{r}-\mathbf{R}_{\alpha}(t)\right|}+\frac{Z_{1} \alpha}{r}+\frac{i \mu \omega}{M_{2}} \frac{d \mathbf{R}_{\alpha}(t)}{d t} \cdot \mathbf{r}\right],
$$

where $Z_{1}$ is the charge of the alpha particle, $M_{2}$ the mass of the daughter nucleus, $\omega=E_{f}-E_{i}, \alpha_{3}$ the third of the Dirac matrices, $\beta$ the velocity of the alpha particle relative to $c, \mu=M_{1} M_{2} /\left(M_{1}+M_{2}\right)$ is the reduced mass of the alpha particle, $\Theta(t)$ the Heaviside function, and $r$ and $R_{\alpha}$ are the distances of the electron and alpha particle relative to the daughter nucleus respectively. The presence of the Heaviside function in (3) is necessary as we define $t=0$ to be the moment when the alpha particle appears, and the perturbations are "switched on".

As can be seen from equation (3), the Hamiltonian consists of three terms. The terms represent (i) the operator for the Coulombic interaction between the emerging alpha particle and the electron, (ii) the shake-off term arising as a result of the parent 
nucleus donating a charge of 2 to the escaping alpha particle and (iii) the recoiling motion of the nucleus from the escaping alpha particle $[4,10]$.

Inspection of the Hamiltonian (3) reveals that further evaluation of amplitude (1) demands making a choice as to the particular time dependency of $R_{\alpha}(t)$. We consider here two scenarios of alpha particle motion, i.e. instantaneous attainment of its final velocity and realistic Coulombic repulsion. For the first scenario we set $R_{\alpha}(0)=d^{2} R_{\alpha}(0) / d t^{2}=0, d R_{\alpha}(0) / d t=v$, the "sudden" approximation as per $[7,8]$, and rewrite amplitude (1) in the form

$$
\begin{aligned}
a_{f i}= & -\int_{0}^{\infty} e^{i \omega t}\left\langle\psi_{f}\left|\frac{Z_{1} \alpha\left(1-\beta \alpha_{3}\right)}{\left|\mathbf{r}-\mathbf{R}_{\alpha}(t)\right|}\right| \psi_{i}\right\rangle d t \\
& +\frac{1}{\omega}\left\langle\psi_{f}\left|\frac{Z_{1} \alpha}{r}\right| \psi_{i}\right\rangle \\
& +i\left\langle\psi_{f}\left|\frac{\mu \mathbf{v} \cdot \mathbf{r}}{M_{2}}\right| \psi_{i}\right\rangle .
\end{aligned}
$$

Alternatively, one may make the more physically accurate assumption that the alpha particle, instead of reaching its final velocity instantaneously at $t=0$, appears at a particular distance outside of the nucleus (having tunnelled through the potential barrier) and achieves its final velocity as a result of Coulombic repulsion from the daughter nucleus. The equation of motion used to describe this situation is [2]

$$
\dot{R}_{\alpha}(t)=\sqrt{\frac{-2 Z_{1} Z_{2} \alpha}{\mu R_{\alpha}(t)}+v_{f}}, \quad R_{\alpha}(0)=\frac{2 Z_{1} Z_{2} \alpha}{\mu v_{f}^{2}},
$$

where $v_{f}$ is the velocity of the ejected alpha particle at $t=\infty$ and $Z_{2}$ is the charge of the daughter nucleus. The initial condition on $R_{\alpha}$ reflects the fact that high energy alpha particles, escaping from the nucleus will not have to tunnel as far as lower energy particles to overcome the potential barrier. For this reason there is an inverse final velocity dependence of the initial condition on $R_{\alpha}$ in equation (5).

By inserting equation (5) into the Hamiltonian, we may write amplitude (1) as:

$$
\begin{aligned}
a_{f i}= & -\int_{0}^{\infty} e^{i \omega t}\left\langle\psi_{f}\left|\frac{Z_{1} \alpha\left(1-\beta \alpha_{3}\right)}{\left|\mathbf{r}-\mathbf{R}_{\alpha}(t)\right|}\right| \psi_{i}\right\rangle d t \\
& +\frac{1}{\omega}\left\langle\psi_{f}\left|\frac{Z_{1} \alpha}{r}\right| \psi_{i}\right\rangle \\
& +i \int_{0}^{\infty} e^{i \omega t}\left\langle\psi_{f}\left|\frac{\mu}{M_{2}} \frac{d^{2} \mathbf{R}_{\alpha}(t)}{d t^{2}} \cdot \mathbf{r}\right| \psi_{i}\right\rangle d t
\end{aligned}
$$


2.2. Evaluation of the matrix elements: Instantaneous attainment of alpha particle velocity

We have shown in the previous section how one obtains the transition amplitude (4) under the "sudden" approximation of alpha particle motion. We will discuss now the further evaluation and simplification of the two terms therein.

The first matrix element of equation (4) is best treated by taking the Fourier transform of $\left\langle\psi_{f}\left|Z_{1} \alpha\left(1-\beta \alpha_{3}\right) /\right| \mathbf{r}-\mathbf{R}_{\alpha}(t) \| \psi_{i}\right\rangle$ immediately [11]

$$
\begin{aligned}
a_{\mathbf{k} \mu_{s}, n \kappa m_{j}}^{1}= & \int_{-\infty}^{\infty} e^{i\left(E_{f}-E_{i}\right) t} \Theta(t)\left\langle\psi_{f}\left|\frac{Z_{1} \alpha\left(1-\beta \alpha_{3}\right)}{\left|\mathbf{r}-\mathbf{R}_{\alpha}(t)\right|}\right| \psi_{i}\right\rangle d t \\
= & 8 \pi Z_{1} \alpha \sum_{L, M, \varkappa} i^{L-M} \int_{0}^{\infty} \frac{s}{s^{2}-(\Delta E)^{2} \beta} \\
& \times\left\langle\psi_{f}\left|\left(1-\beta \alpha_{3}\right) j_{L}(s r) Y_{L}^{M}(\mathbf{r})\right| \psi_{i}\right\rangle B(L, s, \beta, \omega) d s
\end{aligned}
$$

where $B(L, s, \beta, \omega)$ may be expressed as

$$
\begin{aligned}
B(L, s, \beta, \omega)= & -\frac{1}{\pi \omega}\left[(-i)^{L-1} 2^{-L-2} s \sqrt{(2 L+1)} \Gamma(L+1)\left(-\frac{s^{2} \beta^{2}}{\omega^{2}}\right)^{L / 2}\right. \\
& \left.\times{ }_{2} \tilde{F}_{1}\left(\frac{L+1}{2}, \frac{L+2}{2} ; L+\frac{3}{2} ; \frac{s^{2} \beta^{2}}{\omega^{2}}\right)\right]
\end{aligned}
$$

The further computation of the matrix element $\left\langle\psi_{f}\left|\left(1-\beta \alpha_{3}\right) j_{L}(s r) Y_{L}^{M}(\mathbf{r})\right| \psi_{i}\right\rangle$ has been discussed in [11] in equations (7)-(9). The presence of the time dependent Heaviside function as part of the subject of the Fourier transform accounts for the different appearance of $B$ in comparison to that shown elsewhere $[11,12]$.

The matrix element of the shake-off operator in equation (4) has a simpler representation

$$
\begin{aligned}
a_{\mathbf{k} \mu_{s}, n \kappa m_{j}}^{2}= & \alpha Z_{1} i^{l+1}\left(l, m_{j}-\mu_{s}, \frac{1}{2}, \mu_{s} \mid j, m_{j}\right) e^{-i \Delta_{\kappa}} Y_{l}^{m_{j}-\mu_{s} *}(\hat{\mathbf{k}}) \delta_{m_{j}, \mu_{\jmath}} \\
& \times\left(\int_{0}^{\infty} g_{E, \kappa}(r) g_{n, \kappa}(r) r d r+\int_{0}^{\infty} f_{E, \kappa}(r) f_{n, \kappa}(r) r d r\right),
\end{aligned}
$$

where $f_{n, \kappa}(r), g_{n, \kappa}(r)$ and $f_{E, \kappa}(r), g_{E, \kappa}(r)$ represent the small and large radial components of $\left|\psi_{i}\right\rangle$ and $\left|\psi_{f}\right\rangle$ respectively. The spherical symmetry of the shake-off perturbation restricts the excitation of the electron into a continuum state where $\kappa_{i}=\kappa_{f}$ solely for this perturbation, hence the equivalence of the final and initial quantum state variables in equation (9).

The third term in equation (4), the recoil interaction, may be given by

$$
a_{\mathbf{k} \mu_{s}, n \kappa m_{j}}^{3}=\sum_{\varkappa, \mu_{\jmath}} i^{\ell}\left(\ell, \mu_{\jmath}-\mu_{s}, \frac{1}{2}, \mu_{s} \mid \jmath, \mu_{\jmath}\right) e^{-i \Delta_{\varkappa}} Y_{\ell}^{\mu_{\jmath}-\mu_{s} *}(\hat{\mathbf{k}})
$$




$$
\times \frac{\mu v}{M_{2}} a_{G G F F}^{3, \varkappa, m_{j}}
$$

where $a_{G G F F}^{3, \varkappa, m_{j}}$ can be found in the appendix.

\subsection{Evaluation of the matrix elements: Alpha particle motion under Coulombic repulsion}

In section 2.2 we have treated the transition amplitude under the "sudden" approximation of alpha particle motion. In this section, we will discuss the simplification of the elements of the transition amplitude (6) for the realistic motion of the alpha particle. In this construct, it becomes impossible to express the transition amplitude analytically and is therefore necessary to use numerical techniques within the co-ordinate space representation. In this case, the multipole decomposition of $1 /\left|\mathbf{r}-\mathbf{R}_{\alpha}(t)\right|$ in the treatment of the electron-alpha particle Coulombic interaction is required and is given by

$$
\frac{1}{\left|\mathbf{r}-\mathbf{R}_{\alpha}(t)\right|}=\sum_{L, M} \frac{4 \pi}{2 L+1} \frac{\rho_{<}^{L}(t)}{\rho_{>}^{L+1}(t)} Y_{L}^{M *}(0,0) Y_{L}^{M}(\hat{\mathbf{r}}) \delta_{M, 0},
$$

where $\rho_{<}^{L}(t) / \rho_{>}^{L+1}(t)=\operatorname{Min}\left[\mathrm{r}, \mathrm{R}_{\alpha}(\mathrm{t})\right]^{L} / \operatorname{Max}\left[\mathrm{r}, \mathrm{R}_{\alpha}(\mathrm{t})\right]^{L+1}$. Using this decomposition, the representation of the first term of equation (6), the electron-alpha particle Coulombic interaction $a_{\mathbf{k} \mu_{s}, n \kappa m_{j}}^{1}$, can be written in coordinate space as

$$
\begin{aligned}
a_{\mathbf{k} \mu_{s}, n \kappa m_{j}}^{1}= & -\sum_{\varkappa \mu_{j} L} \int_{0}^{\infty} d t e^{i \Delta E t} Z_{1} \alpha \\
& \times i^{\ell}\left(\ell, \mu_{\jmath}-\mu_{s}, \frac{1}{2}, \mu_{s} \mid \jmath, \mu_{\jmath}\right) e^{-i \Delta_{\varkappa}} Y_{\ell}^{\mu_{\jmath}-\mu_{s} *}(\hat{\mathbf{k}}) \\
& \times\left(a_{G G F F}^{1, \varkappa, L, m_{j}}(t)+a_{G F}^{1, \varkappa, L, m_{j}}(t)+a_{F G}^{1, \varkappa, L, m_{j}}(t)\right),
\end{aligned}
$$

where the formulas for $a_{G G F F}^{1, \varkappa, L, m_{j}}(t), a_{G F}^{1, \varkappa, L, m_{j}}(t), a_{F G}^{1, \varkappa, L, m_{j}}(t)$ may be found in the appendix. The second part of equation (6), the shake-off term, has exactly the same form as that shown in equation (9). Finally, the recoil term of equation (6) may be given by

$$
\begin{aligned}
a_{\mathbf{k} \mu_{s}, n \kappa m_{j}}^{3}= & \sum_{\varkappa, \mu_{\jmath}} \int_{0}^{\infty} d t e^{i \Delta E t} i^{\ell}\left(\ell, \mu_{\jmath}-\mu_{s}, \frac{1}{2}, \mu_{s} \mid \jmath, \mu_{\jmath}\right) e^{-i \Delta_{\varkappa}} Y_{\ell}^{\mu_{\jmath}-\mu_{s} *}(\hat{\mathbf{k}}) \\
& \times \frac{\mu}{M_{2}} \frac{d^{2} R_{\alpha}(t)}{d t^{2}} a_{G G F F}^{3, \varkappa, m_{j}},
\end{aligned}
$$

where again $a_{G G F F}^{3, \varkappa, m_{j}}$ can be found in the appendix. 


\subsection{Cross sections and alignment}

In sections 2.2 and 2.3, we have discussed the evaluation of the transition amplitude (1) using the realistic motion of the alpha particle accounting for the Coulombic repulsion from the daughter nucleus and the "sudden" approximation thereof. In both of these scenarios, the cross section for the excitation of an electron from the $2 \mathrm{p}_{3 / 2}$ initial state into the continuum is given by

$$
\sigma_{m_{j}}=\sum_{\mu_{s}=-1 / 2}^{1 / 2} \int_{0}^{\infty} d E_{f}\left(\int d \Omega\left|a_{f i}\right|^{2}\right) .
$$

This partial cross section is found upon integration of the modulus squared of either amplitude (4) or (6) over all electronic final state energies and emission angles and summed over final spin states as we assume that the emitted electron remains unobserved. Moreover, no summation is conducted over the initial $m_{j}$ sub-states in order to obtain a partial ionisation cross section from a particular sub-shell.

The partial ionisation cross sections are related to the alignment of the residual hole in the $2 \mathrm{p}_{3 / 2}$ state. Once the cross sections have been calculated, the alignment of the residual hole in the $2 \mathrm{p}_{3 / 2}$ state can be written as

$$
A_{20}=\frac{\sigma_{\frac{3}{2}}-\sigma_{\frac{1}{2}}}{\sigma_{\frac{3}{2}}+\sigma_{\frac{1}{2}}} .
$$

The alignment, $A_{20}$ is a measure of the relative population of $m_{j}$ sub-states of the $2 \mathrm{p}_{3 / 2}$ state of the residual atom.

With the help of equations (14) and (15) we can calculate the relativistic forms for the cross sections and alignment of alpha decay induced atomic ionisation. In the following section, we proceed to show our results for the calculation of $A_{20}$ for the ionisation process whereby an electron is ejected out of the $2 \mathrm{p}_{3 / 2}$ bound state of Polonium and Thorium nuclei.

\section{Results and discussion}

Having discussed the theoretical background in the previous section, we can now proceed to the calculation of the alignment of atomic inner shells following the alpha-decay of heavy atoms. We have chosen to calculate alignment (15) for Polonium and Thorium atoms. The choice of Polonium has been made as there exists an extensive body of experimental and theoretical research on this element $[13,1,14,15,16]$. Thorium was chosen in order to examine the dependence of the alignment on the charge of the alpha particle emitter. In order to obtain the cross sections (14) necessary to calculate alignment (15), a particular representation of the electronic wavefunctions and model of the alpha particle motion must be selected. For the wavefunctions, we make use of single particle Dirac wavefunctions generated using a screened potential [17] to account for the dominant part of the electron-electron interaction effects. Screening effects also 

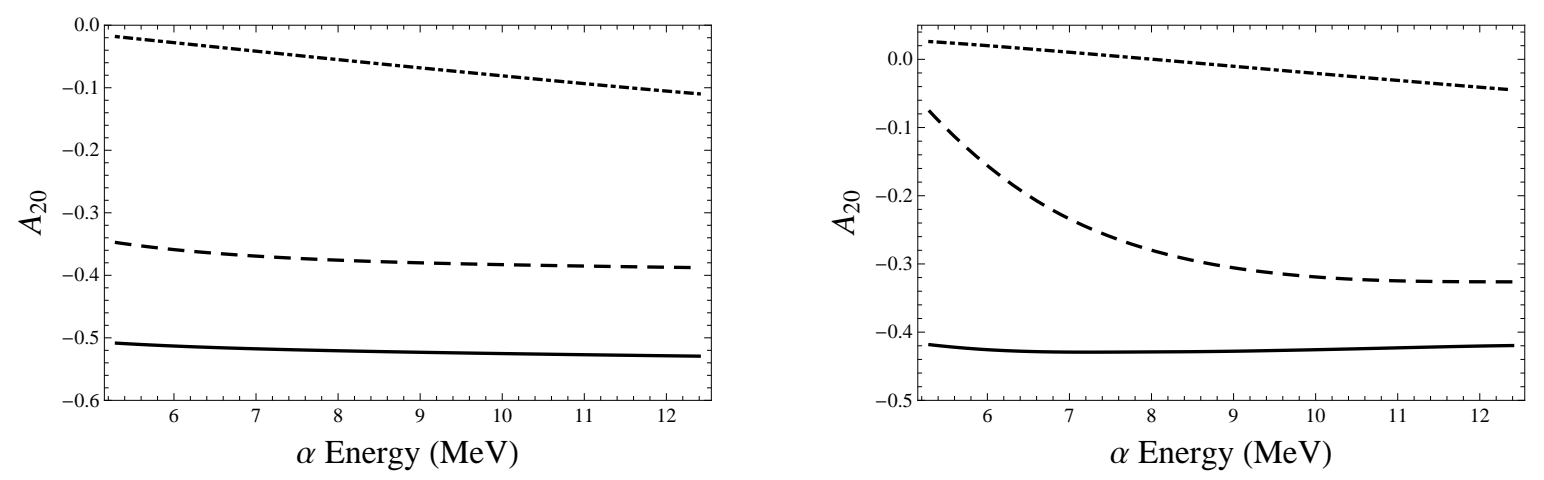

Figure 1. Alignment (15) of the hole created due to ejection of an electron from the $2 \mathrm{p}_{3 / 2}$ shell of Polonium for realistic alpha particle motion (right-panel) and the "sudden" approximation thereof (left-panel). Calculations have been performed using relativistic wavefunctions as generated for screened (solid line) and Dirac Coulomb (dashed line) potentials. Moreover, calculations using screened wavefunctions have been performed excluding shake-off and recoil interactions (dot-dashed line).

cause an energy shift in bound atomic states. This energy shift must be taken into account in the choice of a proper $E_{i}$ in equation (1). The binding energies of the $2 \mathrm{p}_{3 / 2}$ states for neutral Polonium and Thorium are $E_{i}=-13813.8 \mathrm{eV}$ and $E_{i}=-16300.3 \mathrm{eV}$ respectively [18].

In figure 1 we present for both the realistic and "sudden" models of alpha particle motion, our calculations for the alignment of the residual hole created by alpha decay induced excitation of an electron from the $2 \mathrm{p}_{3 / 2}$ state into the continuum of Polonium. These calculations have been performed using screened wavefunctions (solid line) for both models of alpha particle motion. One can see from figure 1 that the alignment (15) is negative and that $\left|A_{20}\right|$ increases slightly with increasing alpha particle velocity. This negative value is a result of certain restrictions placed on the electronic transitions. Namely, from equations (7) and (11) one can see that, given this is a zero impact parameter problem, the total angular momentum projection $m_{j}$ must be conserved during the ionisation process, i.e. $m_{j}=\mu_{\jmath}$. This means that an electron initially bound in a state with $\left|m_{j}\right|=3 / 2$, cannot be excited into either of the $\left|E_{f} \mathrm{~s}_{1 / 2}\right\rangle$ or $\left|E_{f} \mathrm{p}_{1 / 2}\right\rangle$ states. These continuum states constitute a non-negligible contribution to the partial wave summation over $\varkappa$ in equations (7), (10), (12) and (13) and their omission when $m_{j}=3 / 2$ results in a reduction in $\sigma_{3 / 2}$.

After discussing the general characteristics of the alignment shown in figure 1 we are ready now to consider the other effects visible therein. We shall first discuss the effects of screening. In order to demonstrate its importance, we have calculated alignment using wavefunctions generated for both a Dirac Coulomb potential (dashed line) and a screened potential (solid line). As seen from figure 1, screening plays a large role in both the "sudden" and realistic models of alpha particle motion, leading to an overall increase in $\left|A_{20}\right|$. For example, screening effects account for a $43.0 \%$ and $36.6 \%$ increase in the 
magnitude of alignment at alpha particle energies of $6 \mathrm{MeV}$ and $12 \mathrm{MeV}$ respectively in the "sudden" approximation. The reason for this is that the contribution of the electron-alpha particle perturbation to the transition amplitude (1) is more dominant when unscreened wavefunctions are used in its evaluation. The result of this increased contribution from this term is to shift the alignment profile closer to the alignment generated exclusively for the electron-alpha particle perturbation (dot-dashed line).

Apart from the screening effects, a comparison of the left and right panels of figure 1 reveals that calculations for realistic alpha particle motion reduce the modulus of the alignment even further. For instance, the value of $A_{20}$ calculated using screened wavefunctions for realistic alpha particle motion is approxiomately $20 \%$ less over the given spectrum of alpha particle energies than its "sudden" counterpart. This difference may be understood given the fact that higher alpha particle velocities precipitate larger absolute values of alignment. In the "sudden" approximation, the alpha particle attains a constant velocity at $t=0$. For the realistic model however, the alpha particle reaches its final velocity only at $t=\infty$. The velocity of the alpha particle modelled using realistic motion, for equal energies, is always less than in the "sudden" approximation, thus producing the attenuation in alignment shown in the right panel of figure 1.

Having elucidated the role played by screening and alpha particle motion on the alignment (15), it is of interest to understand the interplay between the electronalpha particle interaction and the higher order terms (shake-off and recoil) in the determination of cross sections and hence alignment. Neglecting the comparatively minor contribution of the recoil term, we pursue this discussion with respect to the shake-off and electron-alpha particle interaction perturbations. One can see in figure 1 that the curve representing the alignment calculated neglecting the shake-off and recoil terms (dot-dashed line) suggests weaker alignment than the calculations including all perturbations (solid line). One may expect the opposite, since the shake-off term itself leads to zero alignment. It is known however [4] that inclusion of shake-off in the full transition amplitude (4) or (6) reduces the sum $\sigma_{3 / 2}+\sigma_{1 / 2}$ while the difference in partial cross sections $\sigma_{3 / 2}-\sigma_{1 / 2}$ remains relatively untouched. Therefore the reduction in magnitude of the denominator in equation (15) leads to an increase of the absolute value of alignment as observed in figure 1.

After clarifying the role played by screening, higher order corrections and model dependencies on the alignment of the $2 \mathrm{p}_{3 / 2}$ hole, we can proceed further with a comparison of previous calculations. We have accomplished this in figure 2 where we present our calculations of alignment performed within the "sudden" (dashed line) and realistic (thick solid line) models of alpha particle motion with the predictions of reference [5]. It is evident from this figure that a significant discrepancy exists with the calculations of [5] (thin solid line in figure 2). To reconcile this difference we must discuss the model used in [5]. The results therein were obtained from [6], a study devoted to inner shell ionisation in non-relativistic ion-atom collisions. Disregarding for the moment relativistic shake-off and recoil effects, the transition amplitude in [6] is similar to $a_{f i}$ from equation (1), except that in the current work $a_{f i}$ contains an additional Heaviside 


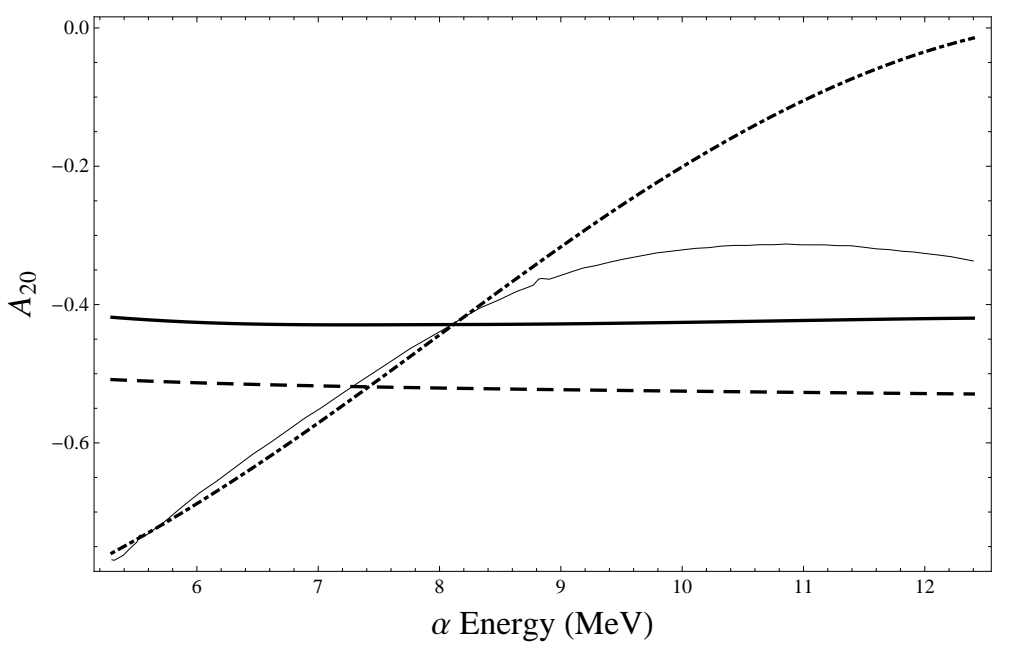

Figure 2. Alignment (15) of the $2 \mathrm{p}_{3 / 2}$ hole calculated by reference [5] (thin solid line) and our replication thereof using relativistic wavefunctions (dot-dashed line). The alignment calculation as shown in figure 1 using screened wavefunctions for the "sudden" (dashed line) and realistic models (solid line) of alpha particle motion for Polonium is presented for comparison.

function. the Heaviside function ensures that unlike in the case of ion-atom collisions, the alpha decay system remains unperturbed before $t=0$, and hence that the integral is conducted over the interval $0 \leq t<\infty$ instead of $-\infty<t<\infty$. To illustrate the role of a proper choice of temporal integration contour, let us restrict ourselves to the dominant time dependent term in equation (1), which for the sake of simplicity is written in the "sudden" approximation as the electron-alpha particle interaction matrix element $f(t)=\left\langle\psi_{f}\left|Z_{1} \alpha\left(1-\beta \alpha_{3}\right) /\right| \mathbf{r}-\mathbf{R}_{\alpha}(t) \| \psi_{i}\right\rangle$. By adopting this notation we can regard equation (1) as the Fourier transform of a product

$$
\mathcal{F}_{\omega}[f(t) \cdot g(t)]=\hat{f}(\omega) * \hat{g}(\omega)=\frac{1}{2 \pi} \int_{-\infty}^{\infty} \hat{f}\left(\omega^{\prime}\right) \hat{g}\left(\omega-\omega^{\prime}\right) d \omega^{\prime},
$$

where $\hat{f}$ and $\hat{g}$ are the Fourier transforms of $f$ and $g$ respectively. Taking $g(t)=\Theta(t)$ and its Fourier transform $\hat{g}(\omega)=\pi(\delta(\omega)-i / \pi \omega)$, equation (16) simplifies to

$$
\mathcal{F}_{\omega}[f(t) \cdot \Theta(t)]=\frac{1}{2} \hat{f}(\omega)-\frac{i}{2 \pi} \int_{-\infty}^{\infty} \frac{\hat{f}\left(\omega^{\prime}\right)}{\left(\omega-\omega^{\prime}\right)} d \omega^{\prime} .
$$

As seen from equation (17), the amplitude for alpha decay induced electronic excitation can be written as half the amplitude of its conventional ion-atom collision equivalent plus a convolution term. In reference [5], only the first term of equation (17) was accounted for. This comprises the vast bulk of the difference between the line of [5] and our work within figure 2. Nonetheless, a replication of the calculations by [5] has been made (dot-dashed line in figure 2), albeit using relativistic wavefunctions.

By way of comparison of our results with the source data from [6] used for the alignment presented in [5], we have included in table 1 a list of total cross sections. 
Table 1. Comparison of electron ejection cross sections due to $5.403 \mathrm{MeV}$ alpha decay of Polonium as determined in [6], [2] and the present work. Rearrangement of equation 8 of [6] elicits the values displayed here. Values are total cross sections, not partial cross-sections of different total angular momentum projections. Cross sections in column 4 were determined using the technique outlined in section 2.2. All values are to the order of $\times 10^{-4}$

\begin{tabular}{llllll}
\hline Atomic-shell & Ref. [6] & Comparison to [6] & Current work & Ref. [2] & Exp.[13] \\
\hline $\mathrm{L}_{I I}+\mathrm{L}_{I I I}$ & 6.32 & 8.54 & 3.04 & 1.01 & 6.03 \\
$\mathrm{~L}_{I}$ & - & - & 2.43 & 1.73 & 3.05 \\
$\mathrm{~L}_{I I}$ & - & - & 1.14 & 0.25 & 2.83 \\
$\mathrm{~L}_{I I I}$ & - & - & 1.90 & 0.76 & 3.20 \\
$\mathrm{~L}$ & - & - & 5.48 & 2.74 & 9.08 \\
\hline
\end{tabular}

Moreover, table 1 also displays the total cross sections in comparison with previously obtained values and experiment, against which our results compare favourably. The value in table 1 on the first line of column 3 was determined using the transition amplitude (7) where $B$ has been modified to account for the necessary collision framework.

Up to this point, only the results of the alignment of the residual $2 \mathrm{p}_{3 / 2}$ hole following alpha decay induced ionisation of a Polonium atom have been scrutinised. It would be instructive to show the alignment dependence on the charge of the alpha emitter. Such a calculation is presented in figure 3 for that of Thorium and compared to the alignment obtained for the Polonium atom. As can be seen in this figure, the alignment for a Thorium emitter is less than that of a Polonium emitter over the indicated alpha particle energy range. The change in alignment of a Polonium emitter compared with a Thorium emitter shifts from -0.426 to -0.342 at $6 \mathrm{MeV}$ and from -0.420 to -0.360 and $12 \mathrm{MeV}$. Such a charge dependence is a consequence of the fact that the alpha particle, in the course of decay, appears at a larger distance and with less velocity in the case of Thorium in comparison to Polonium. Moreover, recognising that using screened wavefunctions or changing the source charge are quasi-analagous processes, one could consider the alignment profile of Polonium in figure 3 as an exaggerated screening type calculation for Thorium and thus expect that the alignment profile for Polonium would suffer less attenuation than that of Thorium. As mentioned before, calculations of alignment using unscreened wavefunctions impart a more dominant contribution to the transition amplitude (1) from the electron-alpha particle interaction, thus shifting the alignment profile toward zero. These properties, a lower instantaneous velocity, an increased $R_{\alpha}(0)$ in equation (5) and a more dominant electron-alpha interaction term result in the attenuation of the alignment profile for Thorium compared with Polonium as seen in figure 3 . 


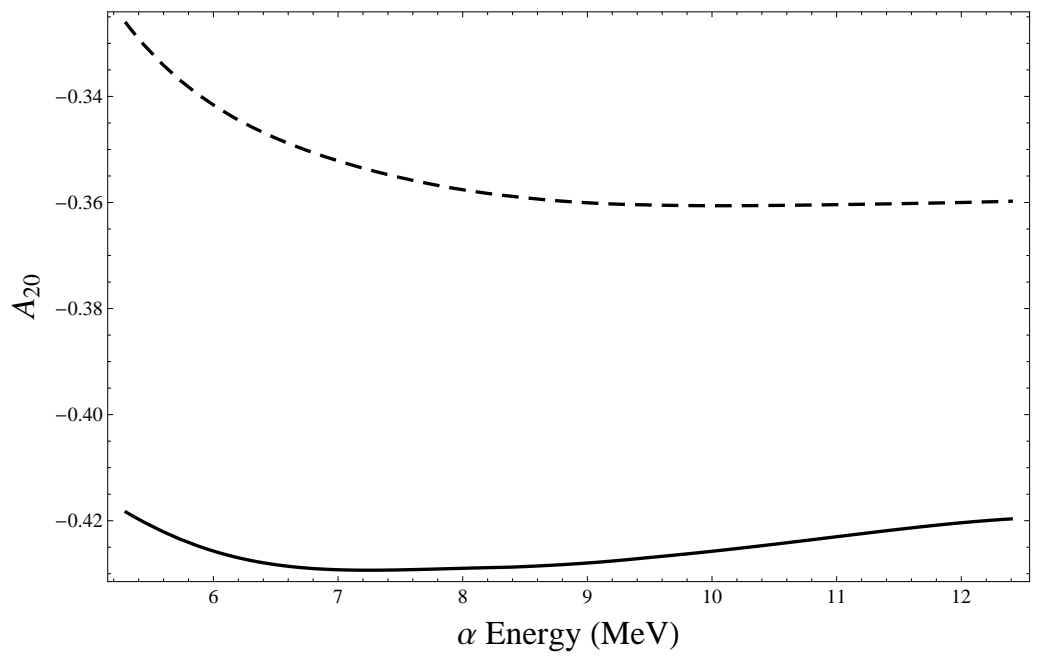

Figure 3. Dependence of the alignment (15) of the $2 \mathrm{p}_{3 / 2}$ hole on the charge of the alpha emitter. The dashed line is the calculation using screened wavefunctions for alignment under the realistic model of alpha particle motion for Thorium and the solid line is its Polonium counterpart.

\section{Summary and conclusion}

In this work we have investigated the excitation into the continuum of electrons of atomic inner shells caused by nuclear alpha decay. Particular attention has been given to the subject of the alignment of the residual hole created by the ejection of an electron from the $2 \mathrm{p}_{3 / 2}$ state of a heavy atom. To understand this process, a full theoretical treatment has been given in section 2 that accounts for shake-off and recoil effects, screened relativistic wavefunctions and the real motion of the alpha particle. We have applied this theory by way of equation (14) to determine alignment values (15) for the process of emission of an electron from $2 \mathrm{p}_{3 / 2}$ shell of Polonium and Thorium atoms. These results have been compared to those presented in [5].

With regard to possible refinements we note that in our theoretical treatment of the escaping alpha particle, we have assumed that it behaves as a classical particle, appearing at a particular distance from the coordinate origin (centred on the daughter nucleus) before moving away from the daughter nucleus under Coulombic repulsion. This neglects the effect the alpha particle has on the atomic shells as it tunnels through the potential barrier confining it originally within the parent nucleus. To compensate for this, one would treat the alpha particle quantum mechanically as per [1]. Further work in this direction is currently underway and will be reported in the near future.

\section{Acknowledgments}

S.M. and A.S. would like to extend their sincerest thanks to Professor Nikolay Kabachnik for his exchange of ideas and opinions in the creation of this work. S.M. acknowledges the support of the International Max Planck Research School - Quantum 
Dynamics (IMPRS-QD). This work is supported by the Helmholtz Gemeinschaft (Nachwuchsgruppe VH-NG-421).

\section{Appendix A. Expansion of matrix elements in co-ordinate space}

Shown in this appendix are the full forms of the matrix elements mentioned in equations (12), (10) and (13). The expanded forms of $a_{G G F F}^{1, \varkappa, L, m_{j}}(t), a_{G F}^{1, \varkappa, L, m_{j}}(t)$ and $a_{F G}^{1, \varkappa, L, m_{j}}(t)$ present in equation (12) are

$$
\begin{aligned}
a_{G G F F}^{1, \varkappa, L, m_{j}}(t)= & W\left(\varkappa, \kappa, L, \mu_{\jmath}, m_{j}\right)\left(\frac{1}{R(t)^{L+1}} \int_{0}^{R(t)} r^{2+L}\left(g_{E, \varkappa}(r) g_{n, \kappa}(r)+f_{E, \varkappa}(r) f_{n, \kappa}\right) d r\right. \\
& \left.+R(t)^{L} \int_{R(t)}^{\infty} r^{1-L}\left(g_{E, \varkappa}(r) g_{n, \kappa}(r)+f_{E, \varkappa}(r) f_{n, \kappa}\right) d r\right), \\
a_{G F}^{1, \varkappa, L, m_{j}}(t)= & W^{\dagger}\left(\varkappa,-\kappa, L, \mu_{\jmath}, m_{j}\right)\left(\frac{1}{R(t)^{L+1}} \int_{0}^{R(t)} r^{2+L}\left(g_{E, \varkappa}(r) f_{n, \kappa}(r)\right) d r\right. \\
& \left.+R(t)^{L} \int_{R(t)}^{\infty} r^{1-L}\left(g_{E, \varkappa}(r) f_{n, \kappa}(r)\right) d r\right), \\
a_{F G}^{1, \varkappa, L, m_{j}}(t)= & -W^{\dagger}\left(-\varkappa, \kappa, L, \mu_{\jmath}, m_{j}\right)\left(\frac{1}{R(t)^{L+1}} \int_{0}^{R(t)} r^{2+L}\left(f_{E, \varkappa}(r) g_{n, \kappa}(r)\right) d r\right. \\
& \left.+R(t)^{L} \int_{R(t)}^{\infty} r^{1-L}\left(f_{E, \varkappa}(r) g_{n, \kappa}(r)\right) d r\right),
\end{aligned}
$$

where

$$
\begin{aligned}
W\left(\varkappa, \kappa, L, \mu_{\jmath}, m_{j}\right)= & \frac{\sqrt{(2 \ell+1)(2 l+1)}}{2 L+1}(l, 0, \ell, 0 \mid L, 0) \\
& \times \sum_{\mu=-\frac{1}{2}}^{\frac{1}{2}}(-1)^{\mu_{\jmath}-\mu}\left(l, m_{j}+\mu, \ell, \mu-\mu_{\jmath} \mid L, 0\right) \\
& \times\left(\ell, \mu_{\jmath}-\mu, \frac{1}{2}, \mu \mid \jmath, \mu_{\jmath}\right)\left(l, m_{j}-\mu, \frac{1}{2}, \mu \mid j, m_{j}\right),
\end{aligned}
$$

and

$$
\begin{aligned}
W^{\dagger}\left(\varkappa, \kappa, L, \mu_{\jmath}, m_{j}\right)= & \beta \frac{\sqrt{(2 \ell+1)(2 l+1)}}{2 L+1}(l, 0, \ell, 0 \mid L, 0) \\
& \times \sum_{\mu=-\frac{1}{2}}^{\frac{1}{2}}(-1)^{\mu_{\jmath}-\mu_{2}} 2 \mu\left(l, m_{j}+\mu, \ell, \mu-\mu_{\jmath} \mid L, 0\right) \\
& \times\left(\ell, \mu_{\jmath}-\mu, \frac{1}{2}, \mu \mid \jmath, \mu_{\jmath}\right)\left(l, m_{j}-\mu, \frac{1}{2}, \mu \mid j, m_{j}\right),
\end{aligned}
$$


the presence of $2 \mu$ in equation (A.5) is due to the fact that $\chi_{\mu^{\prime}}^{\dagger} \hat{\sigma}_{3} \chi_{\mu}=2 \mu \delta_{\mu^{\prime} \mu}$. Furthermore, the expansion of $a_{G G F F}^{3, \varkappa, m_{j}}$ from equation (10) and equation (13) is given by

$$
\begin{aligned}
a_{G G F F}^{3, \varkappa, m_{j}}= & 2 \sqrt{\frac{\pi}{3}}\left(W\left(\varkappa, \kappa, 1, \mu_{\jmath}, m_{j}\right) \int_{0}^{\infty} g_{E, \varkappa}(r) g_{n, \kappa}(r) r^{3} d r\right. \\
& \left.+W\left(-\varkappa,-\kappa, 1, \mu_{\jmath}, m_{j}\right) \int_{0}^{\infty} f_{E, \varkappa}(r) f_{n, \kappa}(r) r^{3} d r\right),
\end{aligned}
$$

\section{References}

[1] Anholt, R. and Amundsen, P. A. 1982 Phys. Rev. A 25(1), 169-177.

[2] Law, J. 1977 Nucl. Phys. A 286(2), 339 - 353.

[3] Revai, J. and Nogami, Y. 1992 Few-Body Systems 13, 75-86.

[4] Blair, J. S. and Anholt, R. 1982 Phys. Rev. A 25(2), 907-920.

[5] Kabachnik, N. M. 1985 J. Phys. B: At., Mol., Phys. 18(13), L423.

[6] Kocbach, L., Hansteen, J. M., and Gundersen, R. 1983 Physica Scripta 27(1), 54.

[7] Rubinson, W. 1963 Phys. Rev. 130(5), 2011-2021.

[8] Levinger, J. S. 1953 Phys. Rev. 90(1), 11-25.

[9] Eichler, J. and Stöhlker, T. 2007 Phys. Rep. 439(1-2), 1-99.

[10] Amundsen, P. A. 1978 J. Phys. B: At., Mol., Opt., Phys. 11(18), 3197.

[11] Valluri, S. R., Becker, U., Grun, N., and Scheid, W. 1984 J. Phys. B: At., Mol., Phys. 17(21), 4359.

[12] Kocbach, L. 1976 Z. Phys. A., Had., Nucl., 279, 233-236.

[13] Fischbeck, H. J. and Freedman, M. S. 1975 Phys. Rev. Lett. 34(4), 173-176.

[14] Eremin, N., Strizhov, V., Tulinov, A., and Uljanova, O. 1987 Il Nuovo Cimento A (1971-1996) 97, 629-632.

[15] Giardina, G., Fazio, G., Mandaglio, G., Manganaro, M., Sacc, C., Eremin, N., Paskhalov, A., Smirnov, D., Maydanyuk, S., and Olkhovsky, V. 2008 Euro. Phys. J., A - Hadr., Nucl. 36, $31-36$.

[16] Ciocchetti, G. and Molinari, A. 1965 Il Nuovo Cimento B (1965-1970) 40, 69-86.

[17] Salvat, F., Martinez, J. D., Mayol, R., and Parellada, J. Jul 1987 Phys. Rev. A 36(2), 467-474.

[18] Bearden, J. A. and Burr, A. F. 1967 Rev. Mod. Phys. 39(1), 125-142. 